\title{
2014 Canadian Association of Gastroenterology Educational Needs Assessment Report
}

\author{
Kevin Waschke MD, VP CAG Education Affairs, Canadian Association of Gastroenterology
}

$\mathrm{T}$ he annual survey of Canadian Association of Gastroenterology (CAG) members' educational needs was conducted via an online survey sent to 1145 CAG members during March. A total of 248 individuals responded to the survey, of which 218 went on to rate educational topics. Similar to previous years, inflammatory bowel disease (IBD) topics were most in demand for future educational events. Other highly rated areas were: endoscopic techniques and live endoscopy, celiac disease, non-IBD intestinal disorders, gastrointestinal infections, celiac disease and irritable bowel syndrome.

\section{INTRODUCTION}

The purpose of the CAG needs assessment was to provide guidance to the Executive and CAG Education Affairs on areas of greatest educational need. Conducting a needs assessment is a requirement for accreditation of educational events in accordance with the Royal College of Physicians and Surgeons of Canada.

\section{METHODS}

The members of Education Affairs 2014 include Drs Robert Berger, Herbert Brill, Maria Cino, Jamie Gregor, Samir Grover, Phillip Hassard, Gilaad Kaplan, Charles Menard, Maitreyi Raman, Connie Switzer, Kevin Waschke, Elena Verdu, Clarence Wong, Winnie Wong, and trainee members Nauzer Forbes and Catharine Walsh. A subgroup of the committee designed the needs assessment survey, which was a modified version of that used in 2013.

The needs assessment was posted online and members were requested by e-mail to complete the simple 'tick box' survey. Data were compiled and analyzed at the CAG National Office.

The survey collected basic demographic information and examined interest in topics for educational events. Respondents were asked to rate their interest in 58 potential topics for educational events using a five-point scale of no interest-minor interest-neutral/not sure-some interest-very interested. The survey also explored use of CAG continuing professional development (CPD) tools.

\section{RESULTS}

A personalized e-mail request sent to CAG members in early March drew 248 respondents, of whom 218 completed the full survey.

\section{Demographics}

Virtually all respondents (99\%) were CAG members while 7\% were CASL members and $1 \%$ were neither. Sixty-seven percent were male; regarding education, $67 \%$ held an MD or equivalent degree, $31 \%$ and $18 \%$ held a $\mathrm{PhD}$ or MSc, respectively, while $8 \%$ held another degree. Of the 176 respondents for whom the question was applicable the year of medical school graduation was before 1980 for $12 \%, 1980$ to 1989 for $11 \%, 1990$ to 1999 for $12 \%, 2000$ to 2004 for $14 \%$ and 2005 or later for $23 \%$. For the 189 respondents for whom the question was applicable, the majority were predominantly teaching-hospital based (77\%), rather than being community-based with $(20 \%)$ or without (3\%) hospital privileges.

Most replies were from individuals in Ontario (37\%), followed by Alberta (24\%), Quebec (15\%) and British Columbia (9\%). Responses were distributed roughly in proportion to provincial population with the exception of a strong showing from Alberta, and slightly fewer respondents from Quebec.

Respondents' specialty was identified as adult gastroenterology by $40 \%$, pediatric gastroenterology by $7 \%$ and hepatology by $2 \%$. Basic and clinical scientists made up $26 \%$ and $2 \%$, respectively, of respondents. Residents and fellows accounted for $17 \%$, and 'Other' roles for $7 \%$.

Regarding where respondents spend their time, $47 \%$ identified clinical practice as their primary focus and $35 \%$ noted basic research ( $>50 \%$ research). Clinical research $(>50 \%)$ and clinician-researchers ( $\leq 50 \%$ research) formed the next biggest group at $6 \%$ each. Less commonly individuals were clinician-teachers $(\leq 50 \%$ teaching) $(4 \%)$, or were involved in administration (>50\%) $(1 \%)$ or 'other' duties $(2 \%)$.

\begin{abstract}
Educational topics
The percentage of respondents who were 'Very interested' in each topic are shown in Figures 1 to 4 for the 58 educational topics surveyed. Consistent with past years, IBD topics remain extremely popular; in addition endoscopic techniques, live endoscopy, non-IBD intestinal disorders, approach to gastrointestinal infections, celiac disease, and irritable bowel syndrome (IBS) were among the most desired educational areas (Figure 1). When examined by various demographic splits (adult vs. pediatric gastroenterologist, basic scientists, teaching hospital vs. community hospital respondents) the most-desired topics ranked somewhat differently (Table 1 ). For a basic science symposium the topics most in demand (percent very interested) were microbiome (43\%) followed by mechanisms of inflammation in colitis (36\%), mechanisms of disease in IBS (30\%) and understanding autoimmunity in relation to gut disease $(27 \%)$.
\end{abstract}

\section{CPD tools}

With respect to CAG CPD tools used over the past two years the one noted to significantly increase knowledge was by far Canadian Digestive Diseases Week (CDDW) (28\%), followed by CAG consensus conference documents (21\%) and CAG Visiting Professor Lectures (19\%). The tools that significantly changed practice again were CDDW (25\%), CAG consensus conference documents (15\%), the Canadian Journal of Gastroenterology $\mathcal{E}$ Hepatology (14\%) and CAG co-developed programs (13\%).

\section{DISCUSSION}

Twenty-two percent of the solicited membership participated in the survey. An ongoing priority for Education Affairs is to develop innovative and easy assessment tools to encourage greater participation by members in order to accurately reflect their educational needs.

IBD remains the highest priority of respondents despite yearly CDDW sessions in this area since 2002. Apart from IBD, endoscopic techniques and live endoscopy, non-IBD intestinal disorders, gastrointestinal infections, celiac disease, and irritable bowel syndrome were popular. CAG Education Affairs is actively working to increase the quantity and quality of educational materials and programs that members can utilize as part of their ongoing maintenance of certification activities. These findings, along with evaluations of CDDW 2014 and identification of unrecognized educational needs, will form the basis of the 2015 CDDW program.

\section{The CAG is proud to acknowledge its Benefactor Corporate Sponsors:}

AbbVie Corporation

Pentax Canada Inc
Olympus Canada Inc

Janssen Inc

Takeda Canada Inc 


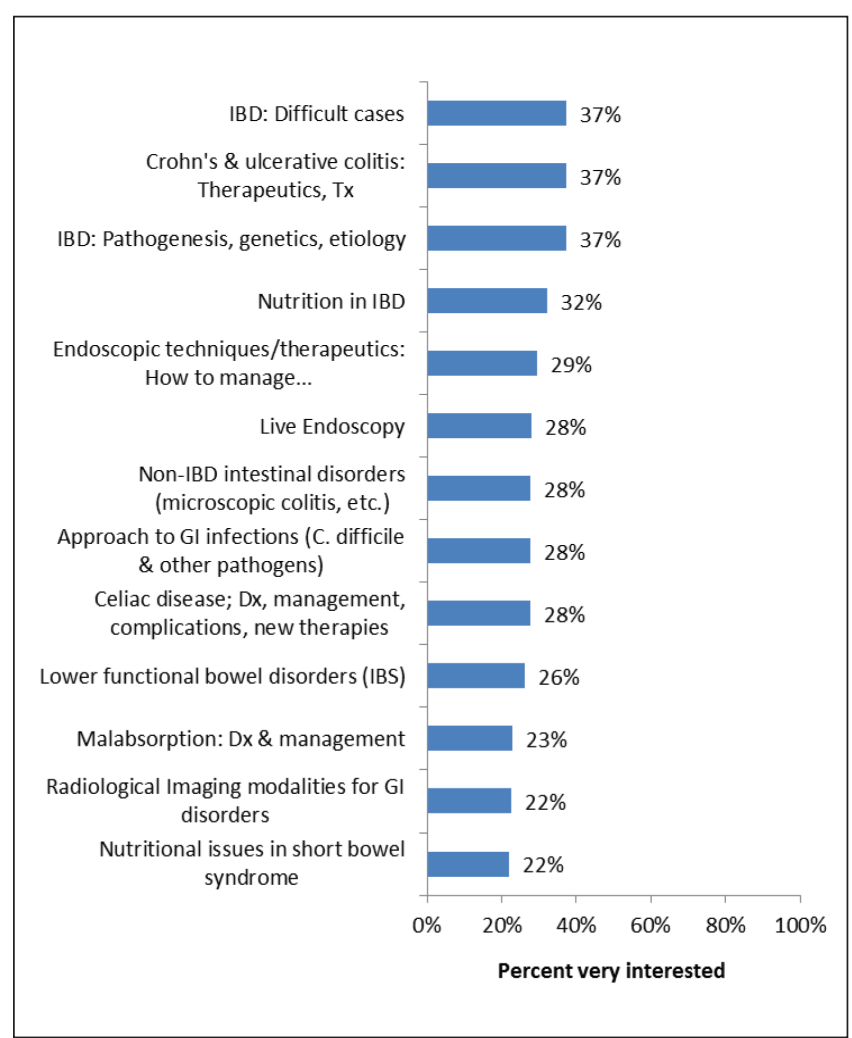

Figure 1) The 13 most popular topics for educational events based on the percent of respondents who were 'very interested' in the area. C difficile Clostridium difficile; Crohn's Crohn disease; Dx Diagnosis; GI Gastrointestinal; IBD Inflammatory bowel disease; IBS Irritable bowel syndrome; Tx Treatment

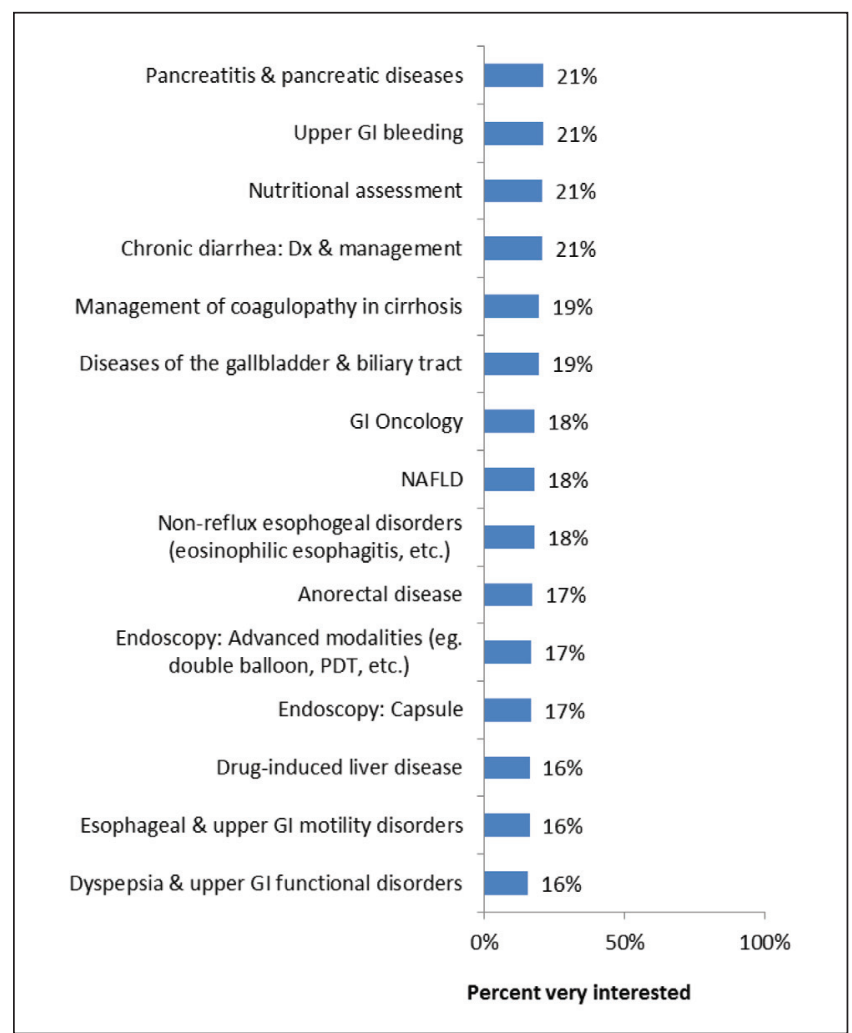

Figure 2) Educational topics in which $16 \%$ to $21 \%$ of respondents were 'very interested'. Dx Diagnosis; GI Gastrointestinal; NAFLD Nonalcoholic fatty liver disease; PDT Photodynamic therapy

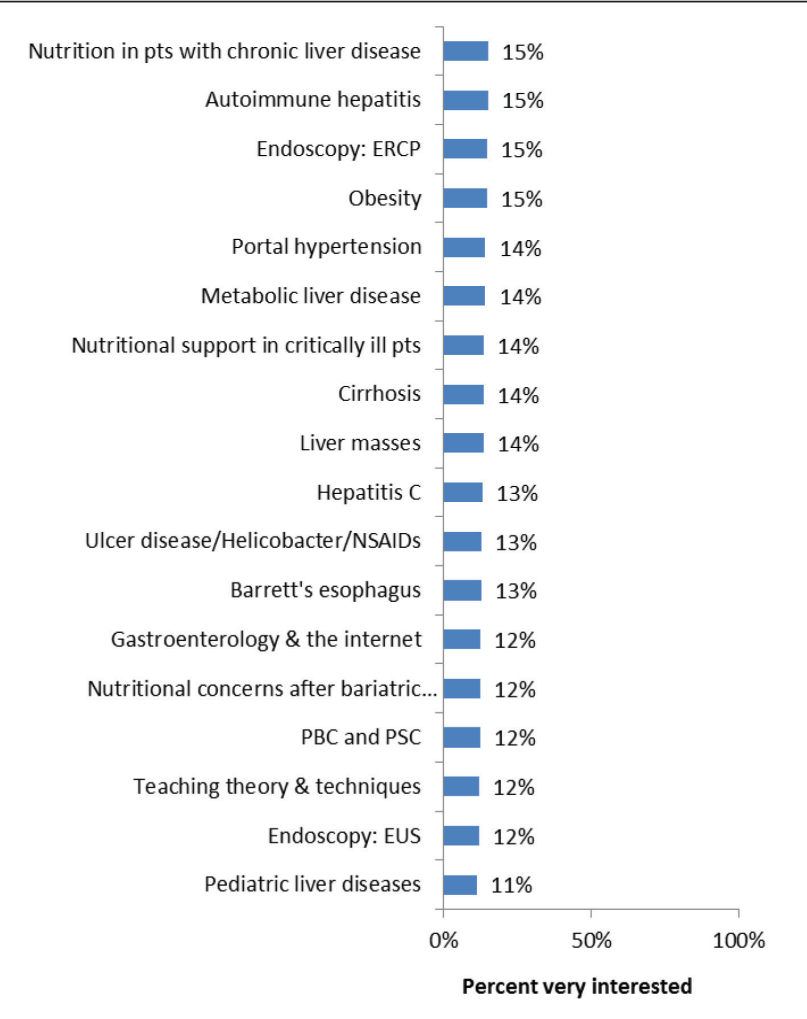

Figure 3) Educational topics in which $11 \%$ to $15 \%$ of respondents were 'very interested'. ERCP Endoscopic retrograde cholangiopancreotography; Helicobacter Helicobacter pylori; NSAIDs Nonsteroidal anti-inflammatory drugs; EUS Endoscopic ultrasound; PBC Primary biliary cirrhosis; PSC Primary sclerosing cholangitis; pts Patients

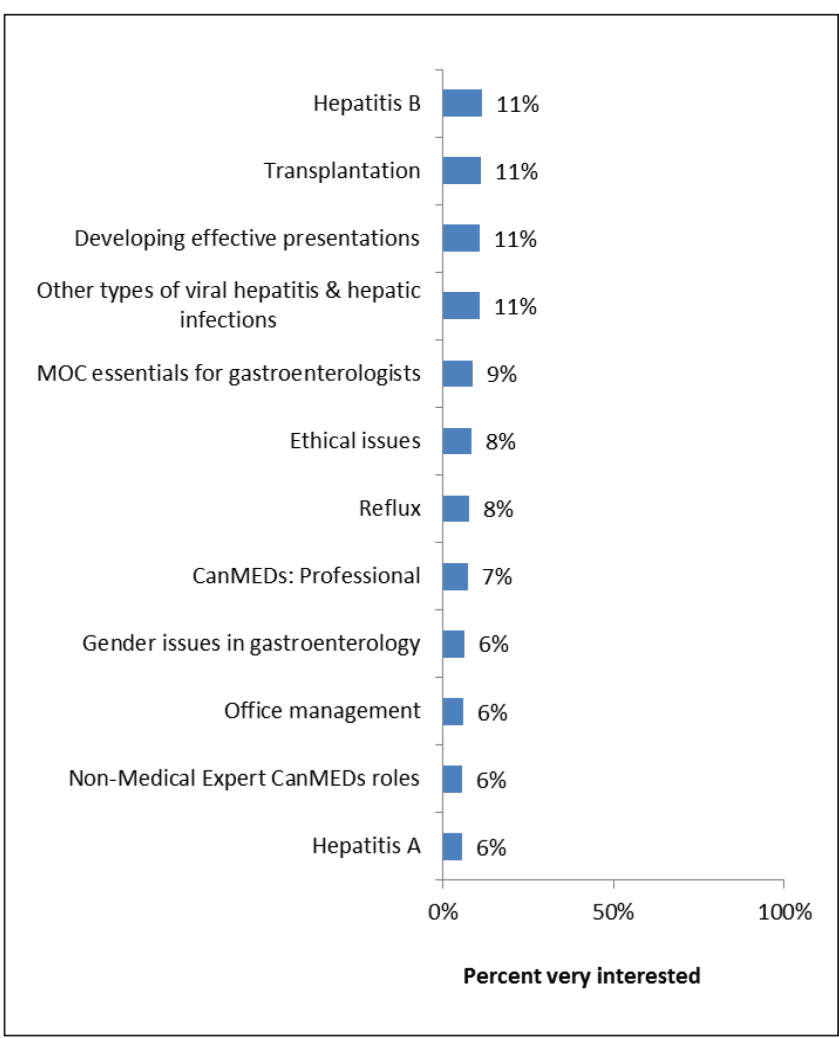

Figure 4) Educational topics in which 6\% to $11 \%$ of respondents were 'very interested' 
TABLE 1

Most popular educational topics according to respondent subgroup

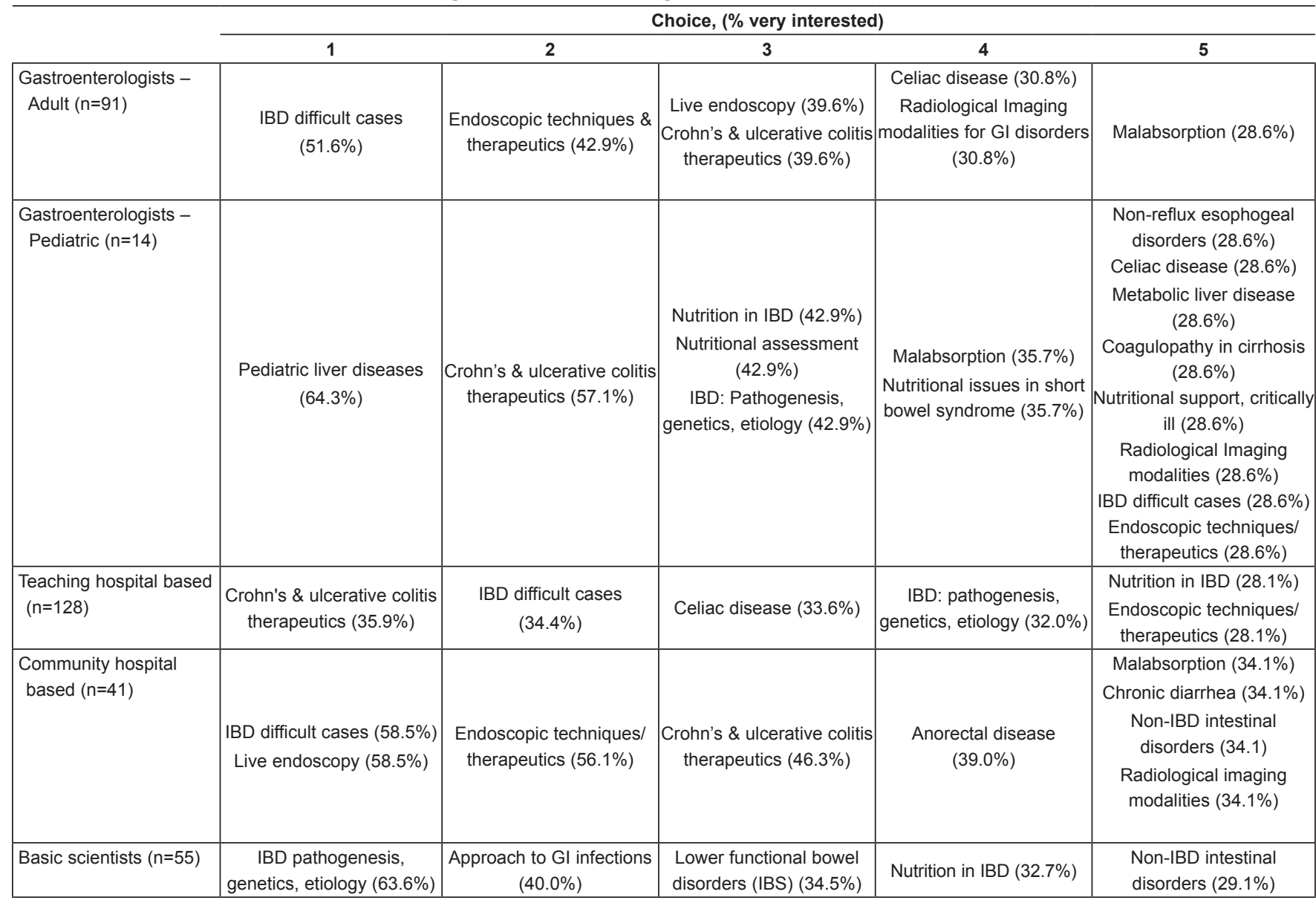

CD Crohn disease; GI Gastrointestinal; IBD Inflammatory bowel disease; IBS Irritable bowel syndrome 


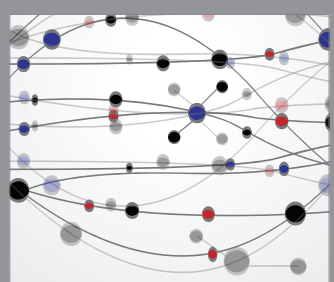

The Scientific World Journal
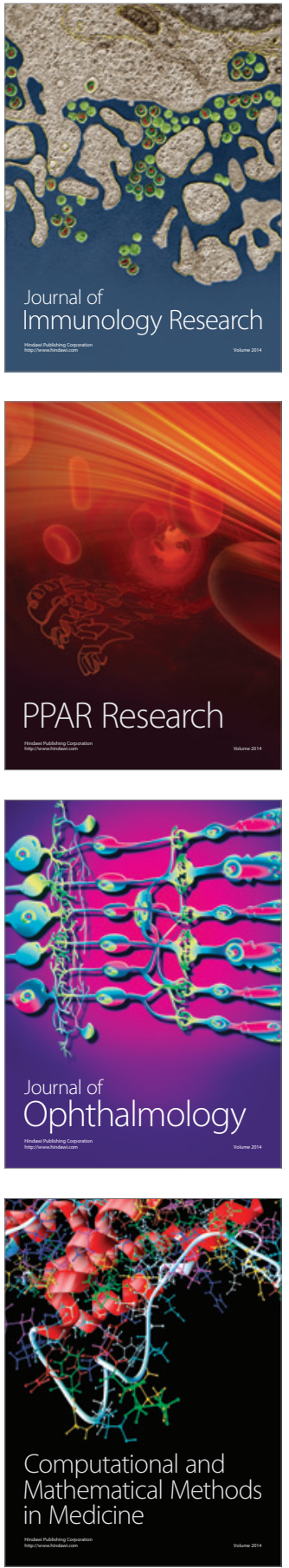

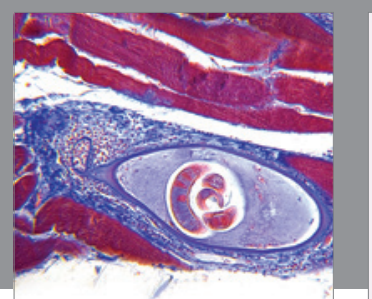

Gastroenterology Research and Practice

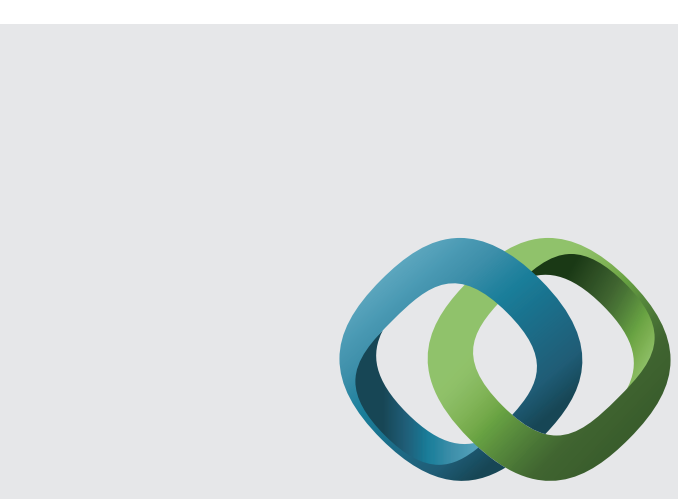

\section{Hindawi}

Submit your manuscripts at

http://www.hindawi.com
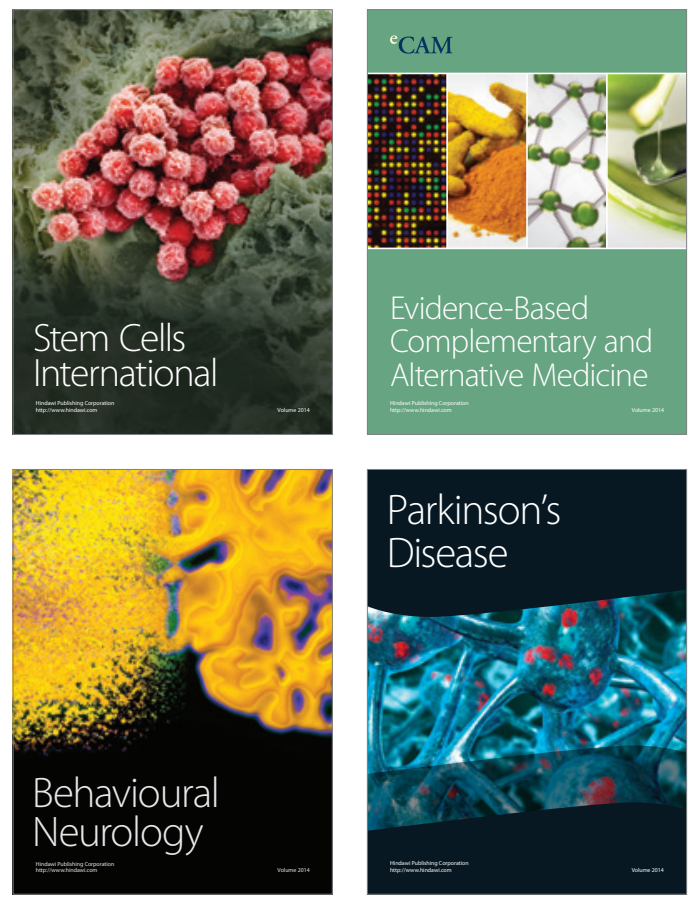
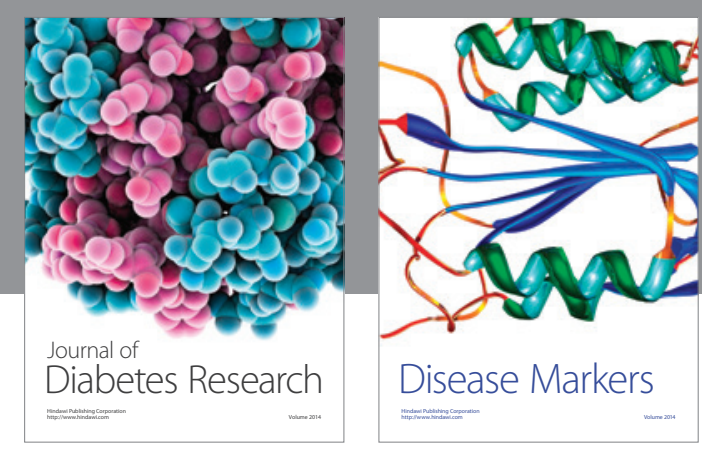

Disease Markers
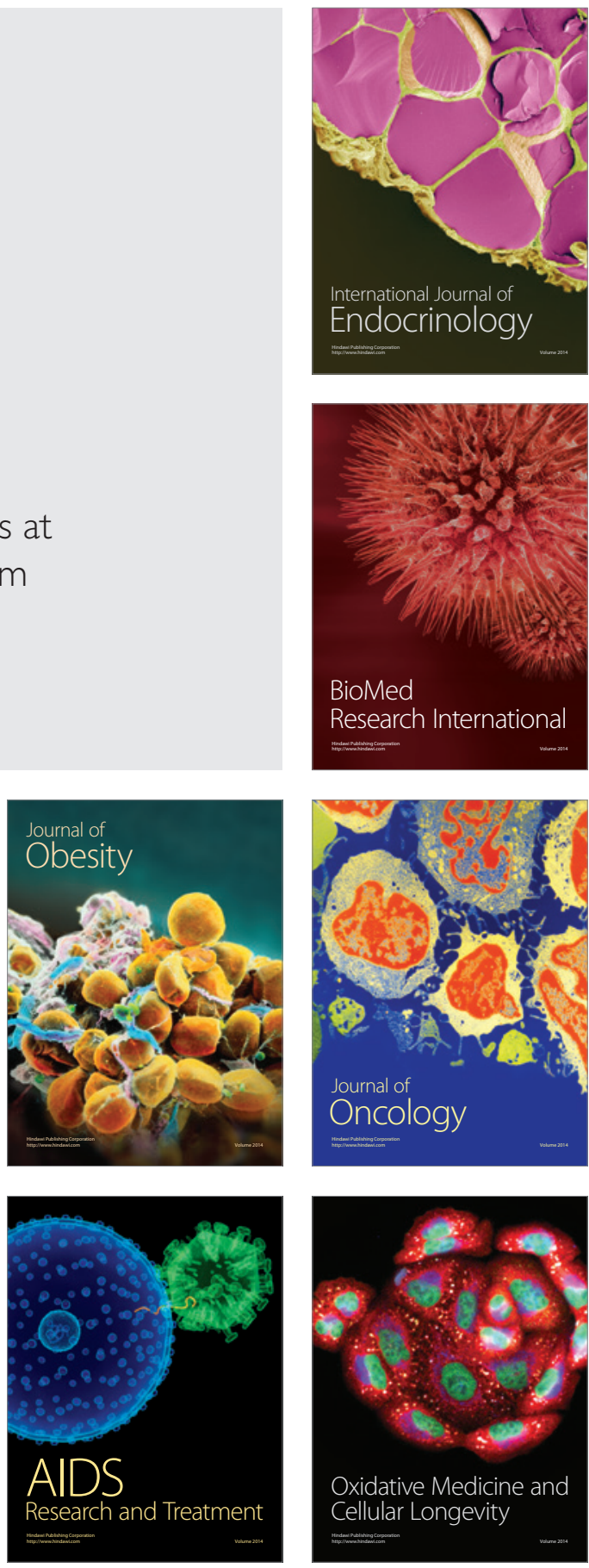